Solid State NMR Measurements for Preliminary Lifetime Assessments in gamma-Irradiated and Thermally Aged Siloxane Elastomers

S. C. Chinn, J. L. Herberg, A. M. Sawvel, R. S. Maxwell

December 3, 2004 
This document was prepared as an account of work sponsored by an agency of the United States Government. Neither the United States Government nor the University of California nor any of their employees, makes any warranty, express or implied, or assumes any legal liability or responsibility for the accuracy, completeness, or usefulness of any information, apparatus, product, or process disclosed, or represents that its use would not infringe privately owned rights. Reference herein to any specific commercial product, process, or service by trade name, trademark, manufacturer, or otherwise, does not necessarily constitute or imply its endorsement, recommendation, or favoring by the United States Government or the University of California. The views and opinions of authors expressed herein do not necessarily state or reflect those of the United States Government or the University of California, and shall not be used for advertising or product endorsement purposes.

This work was performed under the auspices of the U.S. Department of Energy by University of California, Lawrence Livermore National Laboratory under Contract W-7405-Eng-48. 


\title{
Solid State NMR Measurements for Preliminary Lifetime Assessments in $\gamma$-Irradiated and Thermally Aged Siloxane Elastomers
}

\author{
Sarah C. Chinn, ${ }^{1}$ Julie L. Herberg, ${ }^{1}$ April M. Sawvel, ${ }^{2}$ and Robert S. Maxwell ${ }^{1}$ \\ ${ }^{1}$ Chemistry and Materials Science Directorate \\ ${ }^{2}$ Energy and Environment Directorate \\ Lawrence Livermore National Laboratory \\ Livermore, CA 94550 U.S.A.
}

\begin{abstract}
Siloxanes have a wide variety of applications throughout the aerospace industry which take advantage of their exceptional insulating and adhesive properties and general resilience. They also offer a wide range of tailorable engineering properties with changes in composition and filler content. They are, however, subject to degradation in radiatively and thermally harsh environments. We are using solid state nuclear magnetic resonance techniques to investigate changes in network and interfacial structure in siloxane elastomers and their correlations to changes in engineering performance in a series of degraded materials. NMR parameters such as transverse $\left(\mathrm{T}_{2}\right)$ relaxation times, cross relaxation rates, and residual dipolar coupling constants provide excellent probes of changes crosslink density and motional dynamics of the polymers caused by multi-mechanism degradation. The results of NMR studies on aged siloxanes are being used in conjunction with other mechanical tests to provide insight into component failure and degradation kinetics necessary for preliminary lifetime assessments of these materials as well as into the structure-property relationships of the polymers. NMR and MRI results obtained both from high resolution NMR spectrometers as well as low resolution benchtop NMR screening tools will be presented.
\end{abstract}

\section{INTRODUCTION}

The application of solid state nuclear magnetic resonance (NMR) spectroscopy to investigate polymer structure and dynamics is well documented.[1] Correlation of NMR parameters such as transverse $\left(\mathrm{T}_{2}\right)$ relaxation rates and residual dipolar coupling constants with mechanical properties have been used in conjunction with other chemical and mechanical tests such as DSC, GPC, and DMA to determine preliminary service lifetimes in a variety of polymers.[2-4] The variety of degradation mechanisms elastomeric components are subject to in aerospace applications generally lead to competing crosslinking and chain scissioning reactions and numerous methods exist to assess these effects, including solvent swelling, gel-permeation chromatography (GPC), and a variety of mechanical methods such as dynamic mechanical analysis.[5] NMR offers the advantage of being able to probe simultaneously structure and dynamics in situ, in controlled environments, on samples of various shapes. Changes in structure can be measured directly from changes in chemical shifts. In addition, changes in crosslink density can be inferred from the resulting effects on the polymer sequential dynamics directly measurable from the NMR data. Further, multinuclear NMR techniques can provide information about dynamics in both specific domains and the bulk system.

NMR methods have shown significant ability to quantify chemical and network changes occurring during siloxane elastomer degradation.[2-4,6,7] In such materials, residual dipolar 
couplings between proton nuclear spins have been shown to be the result of topological constraints interfering with fast reorientations on the NMR timescale that otherwise would be expected to average homonuclear dipolar couplings to zero. The residual dipolar couplings, in fact, have been shown to be quite sensitive to dynamic and morphological changes.[8-11] The measurement of residual dipolar couplings is possible with standard relaxation time (spin-echo) methods using both standard laboratory instrumentation on excised samples of damaged components and non-destructively on intact components using portable unilateral NMR relaxometers[12-14] and magnetic resonance imaging (MRI).

We are using solid state nuclear magnetic resonance techniques to investigate aging mechanisms and correlations to changes in performance for siloxane elastomers. The characterization of the growth of multiple quantum coherences provides insight into silicone network structure by increasing the selectivity of the NMR experiment to the structure and dynamics most connected to the polymer network.[6,11,15] Unilateral NMR relaxometry with the Bruker Optics NMR MOUSE (MObile Universal Surface Explorer) is used to detect areas of differing crosslink density in damaged silicone parts in an effort to develop a QA/QC protocol to be used in the development of new parts. Finally, MRI experiments are being used to produce spatial maps of crosslink densities, which can be correlated to the overall structural homogeneity of the polymer materials.

\section{EXPERIMENT AND RESULTS}

Samples were cured from Dow Corning 745U silicone fluid cured with Luperox 101, Varox DBPH peroxide curing agent. DC-745U fluid is a proprietary formulation with an unknown network structure. ${ }^{1} \mathrm{H}$ and ${ }^{29} \mathrm{Si}\left\{{ }^{1} \mathrm{H}\right\}$ NMR has determined that the elastomers used in the current study contained $~ 98.5 \%$ dimethyl siloxane monomers, $\sim 1.5 \%$ methyl-phenyl siloxane monomers, and a small amount of vinyl siloxane monomers that are converted to short chain $(\mathrm{N}=4)$ alkyl crosslinking sites. Samples were placed either in $5 \mathrm{~mm}$ NMR tubes or Kapak ${ }^{\circledR}$ SealPAK pouches and either sealed under $\mathrm{N}_{2}$ atmosphere or left exposed to air. The samples were exposed to a ${ }^{60} \mathrm{Co}$ source $(1.2 \mathrm{MeV}, 5000 \mathrm{kGray} /$ hour) for the appropriate time required to achieve a dose range from 0-1000 kGray. For high resolution NMR, the samples were then cut into small $(0.1 \mathrm{~cm} \times 0.1 \mathrm{~cm} \times 0.1 \mathrm{~cm})$ squares and placed in the portion of a $5 \mathrm{~mm}$ NMR tube that would be within the coil volume of the probe.

Solid state NMR experiments were performed at 9.4 T on a Bruker Avance 400 with an operating ${ }^{1} \mathrm{H}$ frequency of $400.10 \mathrm{MHz}$. Multiple quantum NMR experiments were performed using the refocused multiple quantum excitation and reconversion pulse sequence described by Saalwächter et al.[11] Detailed experimental parameters have been published elsewhere.[6] Figure 1 shows the MQ growth curves as a function of radiation dose. The solid lines in Figure 1a show attempts to fit the data to three separate components, each with different residual dipolar couplings (RDCs) representing different levels of crosslink density. In fact, the best fit is obtained with a combination of RDCs, indicating that the polymer is made up of two separate components with different crosslink densities. From this analysis, it was possible to extract both the RDCs and relative mole fractions of the lower CLD network monomer as a function of dose (see Figure 1b and c). The RDC and thus the crosslink density of both components increased as a function of radiation, however the overall population of the lower CLD site decreases with dose. 
(a) Multiple quantum growth curves

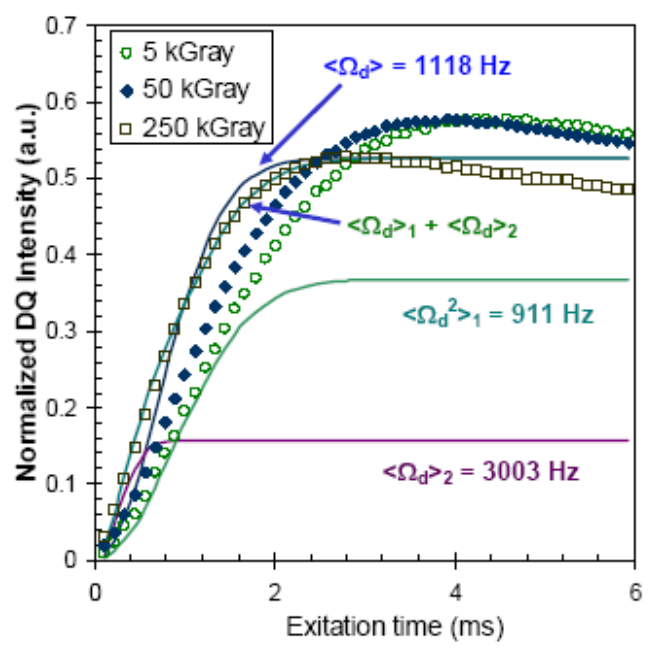

(b) Extracted couplings

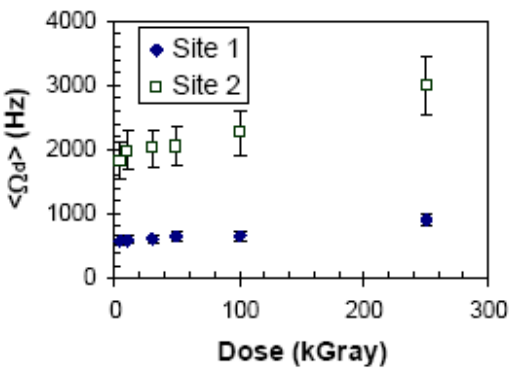

(c) Mole fraction of low CLD component

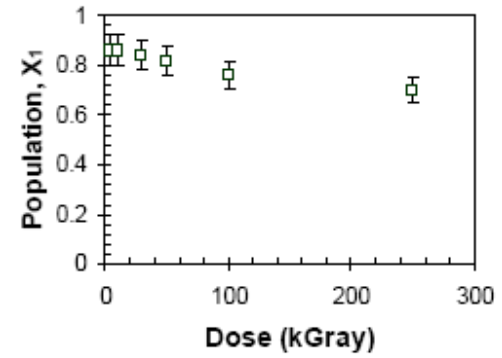

Figure 1. Multiple quantum growth curves (a), extracted RDCs (b), population of low CLD component (c) as a function of radiation.

The wealth of information obtainable with high resolution NMR spectroscopy can also be obtained with inexpensive, benchtop NMR instruments such as the NMR MOUSE. Originally commercialized for the tire industry as an analytical tool for determining defect sites, the MOUSE is a transportable, inexpensive and easy to use system. The MOUSE is currently used to determine mechanical properties of polymers, fat content of dairy products, and is even used in the preservation of artifacts such as historical papers and ancient stone ruins.[16,17] Since the MOUSE enables analysis of intact samples, it is an excellent tool for use in the production of siloxane elastomers, both for rapid analysis of structure-property relationships in new polymer

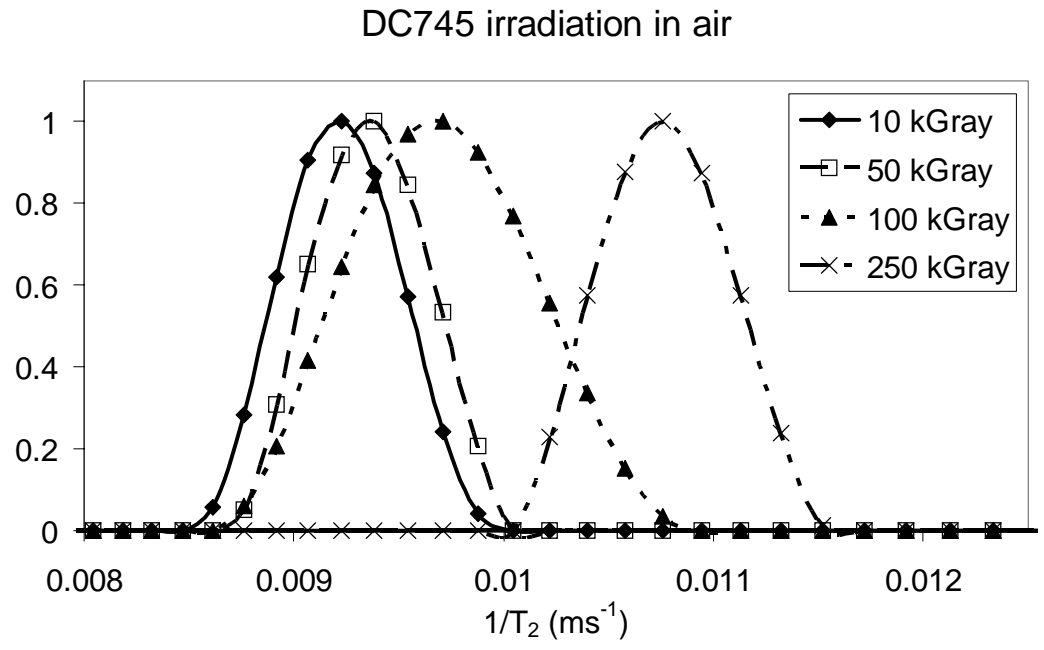

Figure 2. NMR MOUSE results showing the increase in crosslink density as a function of Co-60 irradiation on DC745. 
formulations and as a pre-service screening tool for defect sights.

Since high resolution NMR proved we could observe changes in CLD in irradiated samples, we implemented comparable NMR MOUSE experiments on DC745U. The increase in crosslink density observed in the high resolution experiments can also be seen in simple relaxation measurements obtained with the NMR MOUSE, as seen in Figure 2. Here, samples were placed directly on top of the MOUSE magnet unit for analysis. Static, uniaxial NMR relaxometry experiments were performed using spin-echo decay curves obtained via a CPMG pulse sequence on an NMR MOUSE operating at $17 \mathrm{MHz}$. The experimental parameters were set as follows: echo time of 0.25 ms with 1500 echoes times per experiment and 512 scans signal averaged per echo time. The pulse attenuation, receiver gain, and recycle delay were set to $6 \mathrm{~dB}, 103$, and $1 \mathrm{~s}$, respectively. Decay curves were processed with the Contin application from Bruker Optics, which uses an inverse Laplace transform to yield the distribution of $\mathrm{T}_{2}$ relaxation times.[12] Figure 2 shows the increase in transverse relaxation rate $1 / T_{2}$ as a function of dose. While the crosslink density $\left(1 / T_{2} \propto C L D^{2}\right)$ increases with dose, the results shown here are simply average relaxation values from the bulk system, and the specificity obtained from the multiple quantum NMR experiments above is not provided. We are currently in the process of implementing such pulse sequences with NMR MOUSE analysis.

Aerospace components of these polymers are traditionally produced in large batches and undergo high load in service. It has been our observation that such components are subject to localized permanent deformation in service. We have undertaken an NMR analysis based on the methods outlined above to understand the origin of this degradation. NMR MOUSE analysis was performed on undamaged and damaged sections of a damaged sample. The difference in $\mathrm{T}_{2}$ value (and thus crosslink density) between these regions is clearly detectable in Figure 3. Since the spatial resolution $(\sim 1.5 \mathrm{~cm} \times 1.5 \mathrm{~cm})$ is comparable to the damaged region in the samples, the MOUSE can simply be scanned over the surface of the polymer and the damaged samples can be easily and rapidly identified.

An alternative method for pre-screening the siloxane samples takes advantage of the ability acquire MRI data based on a variety of contrast parameters. In the case of polymer screening, an image can be produced where the intensity of the image is based on the $T_{2}$ or alternatively based on the crosslink density. This essentially provides a spatial map of the crosslink density, and allows for the visible detection of structural heterogeneities or potential defect sites. We used a $T_{2}$ weighted water/fat suppression MRI experiment, which suppresses the fast $T_{2}$ (on the order of $\mu \mathrm{s}$ ) and only gives slower $\mathrm{T}_{2}$ (on the order of ms).[18] The test matrix used was a back to back placement of two samples within the MRI apparatus: a damaged section and pristine section of the siloxane sample. The damaged pad was visibly thinner due to the deformation, but otherwise visually indistinguishable from the undamaged pad. The results of the $2 \mathrm{D} \mathrm{T}_{2}$ weighted water/fat suppression MRI experiment is shown in Figure 4 and was obtained on a Bruker Avance 400 equipped with a microimaging system using an echo time of $10.25 \mathrm{~ms}$, a repetition time of $1 \mathrm{~s}$, and a total time of 4 minutes and 16 seconds. The image was acquired with a Field-of-View (FOV) of $6.0 \mathrm{~cm} \times 6.0 \mathrm{~cm}$ with a resolution of $0.234 \mathrm{~mm} /$ pixel by $0.234 \mathrm{~mm} /$ pixel. The figure verifies that one can distinguish between a damaged section, which has a brighter signal due to the elongated $\mathrm{T}_{2}$ relaxation times, and the pristine section characterized by the fainter signal. 


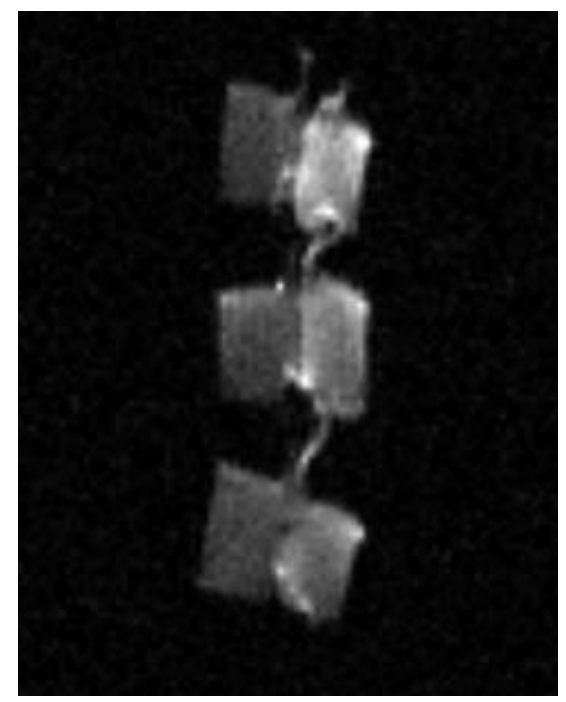

Figure 4. The $2 \mathrm{D} \mathrm{T}_{2}$ weighted water/fat suppression MRI experiments of damaged section and pristine section of DC745. The contrast parameter is $\mathrm{T}_{2}$ relaxation time, with higher signal intensity indicating lower crosslink density.

\section{CONCLUSIONS}

NMR spectroscopy can be used to provide important information about the structureproperty relationships in polymers. Multiple quantum NMR is used to extract information about separate components with different motional properties and detect molecular level changes that result from radiation. In DC745U, radiation-induced crosslinking is observed in both sections with high and low crosslink density, though greater effects are seen in the more crosslinked section. Low resolution unilateral NMR relaxometry measurements obtained with a tabletop NMR MOUSE provide information about the bulk properties of an intact sample and are an ideal tool for screening the polymer throughout the production process. This sensitivity of the MOUSE to changes in crosslink density and residual dipolar couplings allow it to be used in the development of new polymer formulations and as a pre-service screening tool by detecting defects in the polymer which often lead to decreased structural integrity. Additionally, MRI is used to provide a spatial map of the crosslink density throughout excised polymer segments or intact polymers and also can be implemented in the production process.

\section{ACKNOWLEDGEMENTS}

This work was performed under the auspices of the U.S. Department of Energy by the UC Lawrence Livermore National Laboratory under contract \# W-7405-ENG-48.

\section{REFERENCES}

1 K. Schmidt-Rohr and H. W. Spiess, Multidimensional solid state NMR and polymers. (Academic Press, San Diego, 1994).

2 A. Chien, R. S. Maxwell, D. Chambers, B. Balazs, and J. LeMay, J Rad Phys Chem 59, 493 (2000).

3 R. Maxwell and B. Balazs, J Chem Phys 116, 10492 (2002). 
4 R. Maxwell, R. Cohenour, W. Sung, D. Solyom, and M. Patel, Polym Degrad Stab 80, 443 (2003).

5 F. A. Bovey, Effects of ionizing radiation on natural and synthetic high polymers. (Interscience, New York, 1958).

6 R. S. Maxwell, S. C. Chinn, D. Solyom, and R. Cohenour, submitted to Macromolecules (2004).

7 T. Dollase, R. Graf, A. Heuer, and H. W. Spiess, Macromolecules 34, 298 (2001).

8 K. Saalwachter, P. Ziegler, O. Spyckerelle, H. Haider, A. Vidal, and J.-U. Sommer, J. Chem. Phys. 119, 3468 (2003).

9 P. J. Flory, Principles of Polymer Chemistry. (Cornell University Press, Ithaca, NY, 1953).

10 M. Mehring, Principles of High Resolution NMR in Solids. (Springer-Verlag, Berlin, 1983).

11 K. Saalwachter, J. Chem. Phys. 120, 454 (2004).

12 G. Eidmann, R. Savelsberg, P. Blumler, and B. Blumich, Journal of Magnetic Resonance, Series A 122, 104 (1996).

13 A. Guthausen, G. Zimmer, P. Blumler, and B. Blumich, Journal of Magnetic Resonance 130, 1 (1998).

14 A. Wiesmath, C. Filip, D. E. Demco, and B. Blumich, Journal of Magnetic Resonance 149, 258 (2001).

15 R. Fechete, D. E. Demco, and B. Blumich, J. Appl. Poly. Sci. 169, 19 (2004).

16 H. T. Pedersen, S. Ablett, D. R. Martin, M. J. D. Mallett, and S. B. Engelsen, Journal of Magnetic Resonance 165, 49 (2003).

17 B. Blumich, S. Anferova, S. Sharma, A. L. Segre, and C. Federici, Journal of Magnetic Resonance 161, 204 (2003).

18 E. M. Haacke, R. W. Brown, M. R. Thompson, and R. Venkatesan, Magnetic Resonance Imaging: Physical Principles and Sequence Design. (John Wiley \& Sons, New York, 1999). 